\section{Early and Integrated Palliative Care as Valuable Support in Patients With Metastatic Breast Cancer}

\author{
Camelia Rohani, PhD
}

$\mathbf{M}$ etastatic breast cancer (MBC) is the most advanced stage of breast cancer, and refers to cancer that has spread from the breast to other parts of the body. ${ }^{1}$ There were an estimated 2.3 million new cases of female breast cancer and 684,996 death worldwide in $2020{ }^{2}$ with MBC causing the majority of those deaths. $^{3}$ The number of deaths is higher in developing countries compared with developed countries. ${ }^{4}$ The 5 -year survival rate for women with MBC is reported to be $28 \%{ }^{3}$ Around $5 \%$ to $10 \%$ of patients are diagnosed with an advanced stage of disease. ${ }^{5}$ However, early breast cancer can relapse or recur as MBC after a long time following treatments. ${ }^{6}$ There are not enough data, but some studies report that $20 \%$ to $30 \%$ of patients with early breast cancer will develop MBC and will die. ${ }^{6,7}$

Therefore, implementing early integration of outpatient palliative care (OPC) programs is very important. These programs can support patients with advanced cancer, their families, and the healthcare system by reducing unnecessary rehospitalizations and improving the quality of life ( $\mathrm{Q} \circ \mathrm{L}$ ) of patients and their families. ${ }^{8,9}$ The 2016 ASCO palliative care clinical practice guideline recommends integrating palliative care early in the cancer trajectory, together with treatment. ${ }^{10}$ In this issue of JNCCN, Greer et al ${ }^{11}$ present their findings from a valuable clinical trial evaluating the effects of a palliative care intervention on the documentation of end-of-life (EoL) care discussions in the electronic health record; patient-reported discussions about EoL care preferences, QoL, and symptoms of anxiety and depression; and hospice utilization in 2 groups of patients with MBC: a control group $(n=59)$ that received routine care and an intervention group $(n=61)$ that participated in 5 palliative care intervention visits in coordination with their oncologic care. Data collection was done prospectively, at baseline and at 6, 12, 18, and 24 months after baseline and hospice utilization. This type of data collection is very important and provides rich data. The study by Greer et $\mathrm{al}^{11}$ resulted in significant benefits for patients with $M B C$. The rates of discussion and documentation regarding EoL care and hospice services improved within the intervention group compared with the control group. However, study groups did not differ in patient-reported QoL and symptoms of depression and anxiety.

The medical literature highlights positive outcomes for OPC programs around the world, but few details of such programs have been published. ${ }^{8,12}$ Thus, Greer et $\mathrm{al}^{11}$ should be praised for moving forward from designing a palliative care model in their prior studies to applying this specific in patients with MBC. They used an adapted intervention program from their prior research based on evaluation of an integrated palliative and oncology care model. ${ }^{13,14}$ OPC clinics deliver palliative care services on a specific level, such as a short, concise consultation, and coordinate care in alliance with the patient's primary care physician. They also have a referral process. These clinics are also responsible for postdischarge follow-ups, transition of patient care from hospital to home or home healthcare centers, supervision of patient care and medications, and answering questions for patients and their families after hospital discharge. ${ }^{8,9}$

See page 136 for related article.

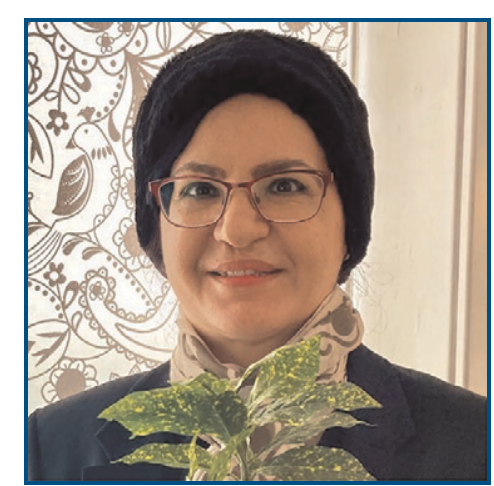

\section{CAMELIA ROHANI, PhD}

Camelia Rohani, $\mathrm{PhD}$, is an affiliated researcher at the Department of Health Care Sciences, Palliative Care Center in Ersta Sköndal Bräcke University College, Campus Ersta, Stockholm, Sweden.

She is also an Associate Professor in the Department of Community Health Nursing in the School of Nursing and Midwifery, Shahid Beheshti University of Medical Sciences, Tehran, Iran.

She is currently working on a project involving integrated home-based palliative care services for patients with cancer in the healthcare system of Iran.

doi: 10.6004/jnccn.2022.7005

The ideas and viewpoints expressed in this commentary are those of the author and do not necessarily represent any policy, position, or program of NCCN. 
There are different models for delivering OPC services that researchers can apply in different healthcare systems, ${ }^{9}$ or the WHO guidelines can be used. ${ }^{15}$ Three models have been introduced successfully in palliative care clinics, including independent, co-located, and embedded. ${ }^{9}$ In the independent model, the OPC clinic is independent from the oncology clinic, and has a specific team, location, administrative support, and referral process. In a co-located OPC clinic, the oncology and palliative clinics are in a common location and patients are visited in coordinated time. In the embedded model, palliative care is delivered in a multidisciplinary oncology clinic that includes the palliative care team, and the patient is visited by both the oncology and palliative care clinicians. Some clinics blend various aspects of these models. ${ }^{9}$

The last issue that should be taken into account is "spirituality" and "spiritual care." In the WHO definition, the spiritual aspect is an integrated part of palliative care. ${ }^{16}$ The spiritual field is a multidimensional area, and patients and caregivers may prefer to apply different terms in place of spirituality, such as meaning of life, philosophy of life, life story, worldview, faith, and religiosity in different countries. ${ }^{17}$ Thus, using the term "existential and spiritual aspects of palliative care" seems more reliable. ${ }^{18}$ Greer et a $1{ }^{11}$ mentioned 5 structured palliative care visits in their program, including visit 1: rapport building; visits 2 to 3 : symptom management, illness understanding, and coping; and visits 4 to 5: treatment decision-making and EoL and advance care planning. Although using existential and spiritual aspects of palliative care is important, it is not obvious in their study. ${ }^{11}$ However, this aspect might have changed the insignificant outcomes of the study. Therefore, it is time to remind clinicians and nurses, when their target group is patients with MBC, that more attention to the existential and spiritual aspects of palliative care is necessary. Providing palliative care specifically for patients with $\mathrm{MBC}$ is important in both palliative care and oncology clinics. Various guidelines ${ }^{19,20}$ are available that can help researchers implement existential and spiritual aspects of palliative care for patients with MBC. Finally, diagnostics for existential crisis in patients with $\mathrm{MBC}$ are recommended, especially in EoL care discussions. This can open a broader area for supportive care and research by focusing on more effective interventions tailored to patients with MBC.

Disclosures: Dr. Rohani has disclosed not having any financial interests, arrangements, or affiliations with the manufacturers of any products discussed in this article or their competitors.

Correspondence: Camelia Rohani, PhD, Ersta Sköndal Bräcke University College, Campus Ersta, Stigbergsgatan 30, Box 11189, SE-100 61,

Stockholm, Sweden. Email: camelia.rohani@esh.se

\section{References}

1. Global Status of Advanced/Metastatic Breast Cancer: 2005-2015 Decade Report, Summary. Accessed January 8, 2022. Available at: https://www.abcglobalalliance.org/pdf/Global-Status-of-mBC-ReportSummary_041416_Digital.pdf

2. Sung H, Ferlay J, Siegel RL, et al. Global Cancer Statistics 2020: GLOBOCAN estimates of incidence and mortality worldwide for 36 cancers in 185 countries. CA Cancer J Clin 2021;71:209-249.

3. Cancer.Net. Breast Cancer - Metastatic: Statistics. Accessed January 8, 2022. Available at: https://www.cancer.net/cancer-types/breast-cancermetastatic/statistics

4. Francies FZ, Hull R, Khanyile R, et al. Breast cancer in low-middle income countries: abnormality in splicing and lack of targeted treatment options. Am J Cancer Res 2020;10:1568-1591.

5. Cardoso F, Costa A, Norton L, et al. 1st International consensus guidelines for advanced breast cancer (ABC 1). Breast 2012;21:242-252.

6. Riggio Al, Varley $\mathrm{KE}$, Welm $\mathrm{AL}$. The lingering mysteries of metastatic recurrence in breast cancer. Br J Cancer 2021;124:13-26.

7. O'Shaughnessy J. Extending survival with chemotherapy in metastatic breast cancer. Oncologist 2005;10(Suppl 3):20-29.

8. Meier DE, Beresford L. Outpatient clinics are a new frontier for palliative care. J Palliat Med 2008;11:823-828.

9. Finlay E, Newport K, Sivendran S, et al. Models of outpatient palliative care clinics for patients with cancer. J Oncol Pract 2019;15:187-193.

10. Ferrell BR, Temel JS, Temin S, et al. Integration of palliative care into standard oncology care: American Society of Clinical Oncology clinical practice guideline update. J Clin Oncol 2017;35:96-112.

11. Greer JA, Beverly M, El-Jawahri A, et al. A randomized trial of a palliative care intervention to improve end-of-life care discussions in patients with metastatic breast cancer. J Natl Compr Canc Netw 2022;20:136-143.

12. Zimmermann C, Ryan S, Hannon B, et al. Team-based outpatient early palliative care: a complex cancer intervention [published online August 12, 2019]. BMJ Support Palliat Care, doi:

13. Hoerger M, Greer JA, Jackson VA, et al. Defining the elements of early palliative care that are associated with patient-reported outcomes and the delivery of end-of-life care. J Clin Oncol 2018;36:1096-1102.

14. Yoong J, Park ER, Greer JA, et al. Early palliative care in advanced lung cancer: a qualitative study. JAMA Intern Med 2013;173:283-290.

15. World Health Organization. Integrating palliative care and symptom relief into primary health care: a WHO guide for planners, implementers and managers. Accessed January 8, 2022. Available at: https://www.who.int/ publications/i/item/integrating-palliative-care-and-symptom-relief-intoprimary-health-care

16. World Health Organization. WHO Definition of Palliative Care. Accessed December, 30, 2018. Available at: http://www.who.int/cancer/palliative/ definition/en

17. Ettema E, Wulp M, Leeuwen Rv, et al. Embedding of the spiritua dimension in palliative consultation services in the Netherlands: inventory, evaluation, and recommendations. Prog Palliat Care 2015; 23:259-266.

18. Existential and Spiritual Aspects of Palliative Care: National guideline. Version 2.0, Accessed January 8, 2022. Available at: https://www. pallialine.nl/uploaded/docs/Zingeving/Existential_and_spiritual_aspects_ of_palliative_care_zonder_linken.pdf?u=1SxZX12018

19. Handzo G, Bowden JM, King S. The evolution of spiritual care in the NCCN Distress Management Guidelines. J Natl Compr Canc Netw 2019; 17:1257-1261.

20. Moosavi S, Borhani F, Akbari ME, et al. Recommendations for spiritual care in cancer patients: a clinical practice guideline for oncology nurses in Iran. Support Care Cancer 2020;28:5381-5395. 